\title{
Hyperlipidemia Management in Proprotein Convertase Subtilisin- Kexin Type 9 (PCSK 9) Inhibitors Era

\author{
Abdulhalim Jamal Kinsara*
}

President of SASE, Director of cardiology fellowship, Ministry of National Guard health Affair. King Abdullah International medical research center, King Saud Bin Abdulaziz University for health sciences, COM-WR

*Corresponding author: Abdulhalim Jamal Kinsara, FRCP, FACC, Associate professor of cardiology, Ministry of National Guard health Affair, King Abdullah research center. King Saud Bin Abdulaziz University for health sciences, COM-WR. Mail code 6599, P.O. Box 9515, Jeddah 21423, Saudi Arabia. Tel 966-12-2266666. Email: akinsara@yahoo.com

\begin{abstract}
Significant improvement in control of cardiovascular risk factors has been driven by performance measurement that focused on attainment of specific risk factor thresholds for blood glucose, lipids and blood pressure. Diet, statin and ezetimibe have reasonably controlled hyperlipidemia. However the discovery of proprotein convertase subtilisin-kexin type 9 inhibitors has given new hope of reaching the target lipid profile especially in the high-risk group and familial hypercholesterolemia. Herewith we reviewed the current data and explore the cardiovascular prevention effect of this group.
\end{abstract}

\section{Keywords}

Proprotein convertase subtilisin-kexin type 9 inhibitors, Evolocumab, Alirocumab, Hyperlipidemia, Familial hypercholesterolemia

Hyperlipidemia is an important risk factor for atherosclerotic cardiovascular disease. Data from 130 VA centers which included 900,000 patients with diabetes, found that $68 \%$ of eligible patients were on statins, half of them were on the need to be on moderate- to high intensity Statins [1].

A meta-analysis of randomized controlled trials showed consistent clinical benefit from decreasing LDL even further in patients with baseline LDL as low as 63 $\mathrm{mg} / \mathrm{dL}$ and for each $38.7 \mathrm{mg} / \mathrm{dL} \mathrm{LDL}$ reduction, $21 \% \mathrm{ma}-$ jor vascular events relative risk reduction were noted. In addition, a linear relationship between lowering serum LDL levels and improving outcomes in cardiovascular disease also noted $[2,3]$.
On the other hand, in spite of this overwhelming data, it was challenged by failure to reach the target with current lipid-lowering drugs. Another challenge was statin intolerance, whether complete, defined as intolerance to any statin at any dose or partial, defined as intolerance to some statin at certain dosage [4].

The target is even more difficult to achieve in Familial Hypercholesterolemia (FH). In PLANET heterozygous $\mathrm{FH}$ registry in the Czech Republic and Slovakia, which was a non-interventional, retrospective and cross-sectional, only $15.4 \%$ of 1755 patients enrolled attained the target LDL value. Fourteen percent (14.0\%) had cardiovascular events in patients attaining the LDL goal, against $86.0 \%$ in the non-target group [5]. Similar data were published in South Africa. The mean untreated and best achieved LDL values during follow up were $8.1 \pm 2.1$ and $4.0 \pm 1.5 \mathrm{mmol} / \mathrm{l}$, respectively [6].

Scientists continued to explore this field till 2003 when proprotein convertase subtilisin-kexin type 9 (PCSK9) was discovered. PCSK9 is a protein mainly secreted by the liver, but a small amount is also secreted from the kidneys and small intestine.

PCSK9 promotes hypercholesterolemia by binding to $L D L$ receptors on the surface of hepatocytes and is transported to lysosomes for degradation. The subsequent reduction in the number of LDL receptors resulted in reduced clearance of LDL from the blood. A less identified mechanism was via the Golgi lysosomal intracellular route [7].

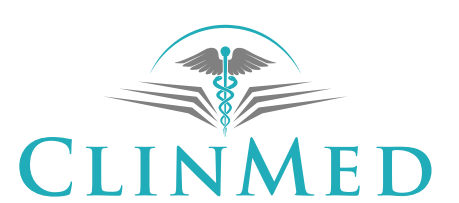

INTERNATIONAL LIBRARY

Citation: Kinsara AJ (2019) Hyperlipidemia Management in Proprotein Convertase Subtilisin-Kexin Type 9 (PCSK 9) Inhibitors Era. Int J Clin Cardiol 6:146. doi.org/10.23937/2378-2951/1410146 Accepted: May 30, 2019; Published: June 01, 2019

Copyright: () 2019 Kinsara AJ. This is an open-access article distributed under the terms of the Creative Commons Attribution License, which permits unrestricted use, distribution, and reproduction in any medium, provided the original author and source are credited. 
The gene located on chromosome $1 p 32.3$ encodes PCSK9. Genetic mutations with gain and loss kept LDLR regulation in a counterbalance. The gene theory was of real interest to researchers. In Global Consortium for studying Lipids Genetics, 32 million variants for association with lipid levels were tested and 118 novel genome-wide significant loci were identified on metaanalysis. A focus on mutations expected to lead to loss of gene function and a phenomena-wide related study, a novel indications for pharmaceutical inhibitors targeting PCSK9 linked to abdominal aortic aneurysm), ANGPTL4 linked to type 2 diabetes and PDE3B linked to triglycerides and coronary disease were suggested [8].

Monoclonal antibodies inhibiting PCSK 9 constitute a new class of lipid lowering drugs. The theoretical advantage became a practical solution with two large placebo-controlled trials evaluating clinical outcomes in patients with LDL $1.8 \mathrm{mmol} / \mathrm{L}$ or greater on maximally tolerated statins and having associated CVD. In the first, evolocumab versus placebo in 27,564 patients over 2.2 years showed a significant reduction in new CVD events (9.8\%, against $11.3 \%)$. The number needed to treat was 67 but there was no difference in death by any cause [9].
In the second study alirocumab was started in 18,924 patients after ACS and followed, up to 2.8 years demonstrating a statistically significant reduction in new CVD events (9.5\% versus $11.1 \%$ ). The number needed to treat was 63 and death by any cause was $3.5 \%$ against $4.1 \%$ [10].

Both drugs showed similar LDL reduction $55 \%$ vs. $53 \%$ and raised HDL $7.6 \%$ vs. $8 \%$ respectively [11]. Significantly more plaque volume reduction was seen with PCSK9. Tolerability was quite high in both studies, with low rates of discontinuation in up to 2.8 years. A reaction at the site of injection was the main adverse side effect. Developing neutralizing antibodies was rare and usually clinically insignificant.

However since data of the long-term effects are still lacking and the drug is a costly one, a step-wise approach is necessary, Figure 1. Dietary interventions remain the golden primary advice. Moderate to high dose statin should been tried. The statin group had a complex approach before being declared a failure or having caused intolerance. Ezetimibe had proved an effective drug with and without statin in preventing cardiovascular event.

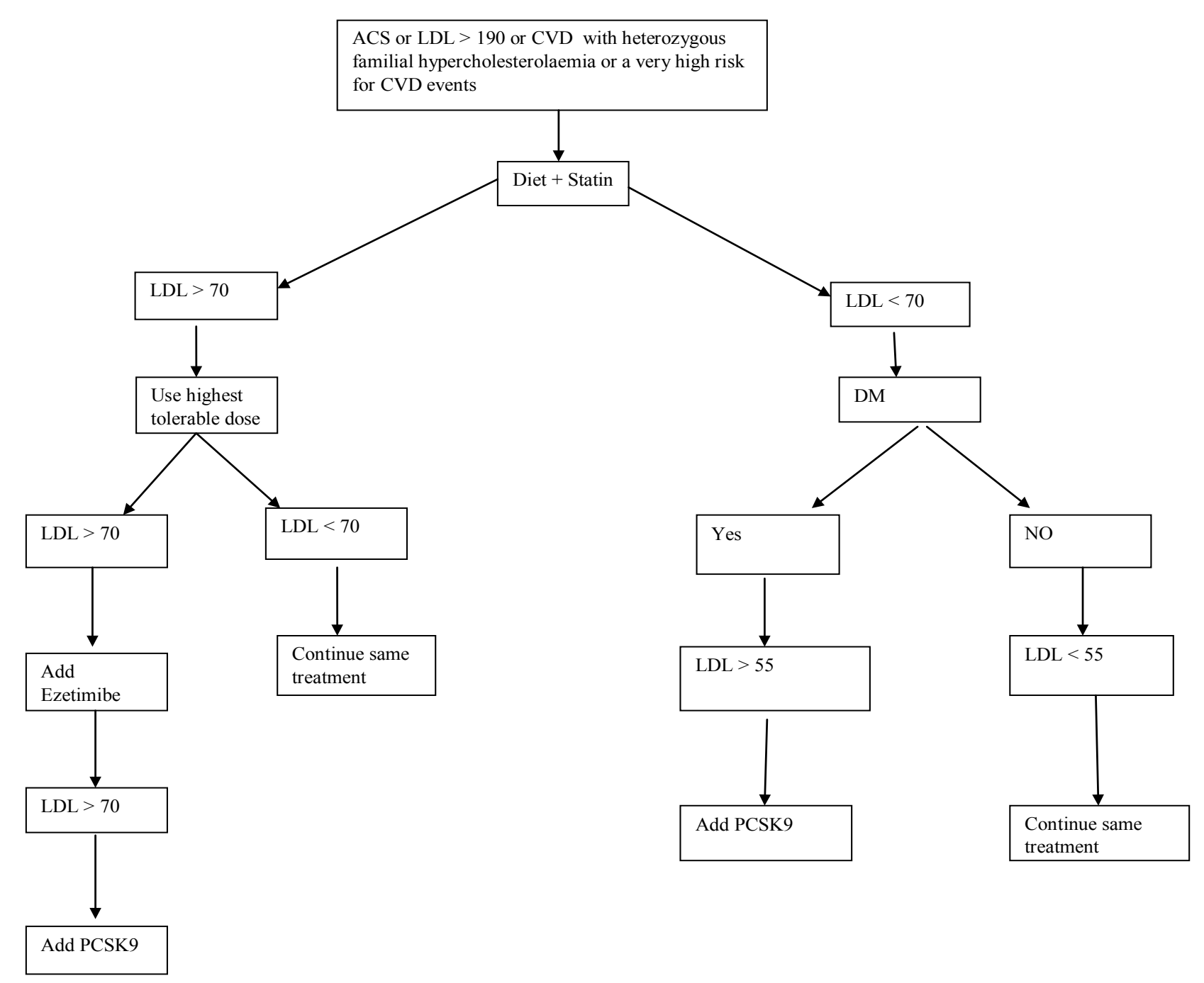

Figure 1: Flow chart. 
If the LDL target is still lacking after a maximal tolerated dose of statin and ezetimibe treatment, then PCSK9 inhibitor would be a safe and effective alternative treatment with good intermediate follow up data [12]. Such a planned approach might change if the current price of the drug be reduced by half [13].

2017 Focused Update of the 2016 ACC Expert Consensus Decision Pathway drew up a map for Decision the Role of Non-Statin drugs to attain the target LDL-Cholesterol in the treatment of Atherosclerotic Cardiovascular Disease Risk. It focused on the very high-risk group, at risk of developing $\mathrm{CV}$ diseases and divided them into uncontrolled ASVCD risk factors compared to controlled risk and categorized age to 18-39 and 40-79-years. The target LDL was set at $70 \mathrm{mg} / \mathrm{dl}$ vs. $100 \mathrm{mg} / \mathrm{dl}$. Familial hypercholesterolemia was considered the highest risk group. Non-HDL was the alternative treatment target.

\section{Conclusion}

PCSK 9 inhibitor is an alternative LDL lowering drug that can either be added or replace statin/ezetimibe to obtain recommended LDL goals in patients with high risk of cardiac event. It has a large safety profile but currently cost-effective in high risk patients.

\section{References}

1. Pokharel Y, Akeroyd JM, Ramsey DJ, Hira RS, Nambi V, et al. (2016) Statin Use and its Facility Level Variation in Patients with Diabetes: Insight from Veterans Affairs National Database. Clin Cardiol 39: 185-191.

2. Navarese EP, Robinson JG, Kowalewski M, Kolodziejczak M, Andreotti F, et al. (2018) Association Between Baseline LDL-C Level and Total and Cardiovascular Mortality After LDL-C Lowering: A Systematic Review and Meta-analysis. JAMA 319: 1566-1579.

3. Shahreyar M, Salem S, Nayyar M, George L, Garg N, et al. (2018) Hyperlipidemia: Management With Proprotein Convertase Subtilisin/Kexin Type 9 (PCSK9) Inhibitors. J Am Board Fam Med 31: 628-634.

4. Zhang H, Plutzky J, Shubina M, Turchin A (2017) Continued statin prescriptions after adverse reactions and patient outcomes: a cohort study. Ann Intern Med 167: 221-227.

5. Vrablik M, Raslová K, Vohnout B, Blaha V, Satny M, et al. (2018) Real-life LDL-C treatment goals achievement in patients with heterozygous familial hypercholesterolemia in the Czech Republic and Slovakia: Results of the PLANET registry. Atherosclerosis 277: 355-361.

6. van Delden XM, Huijgen $\mathrm{R}$, Wolmarans $\mathrm{KH}$, Brice $\mathrm{BC}$, Barron JK, et al. (2018) LDL-cholesterol target achievement in patients with heterozygous familial hypercholesterolemia at Groote Schuur Hospital: Minority at target despite large reductions in LDL-C. Atherosclerosis. 277: 327-333.

7. Cicero AFG, Bove M, Borghi C (2018) Pharmacokinetics, pharmacodynamics and clinical efficacy of non-statin treatments for hypercholesterolemia. Expert Opin Drug Metab Toxicol 14: 9-15.

8. Klarin D, Damrauer SM, Cho K, Sun YV, Teslovich TM, et al. (2018) Genetics of blood lipids among $\sim 300,000$ multiethnic participants of the Million Veteran Program. Nat Genet 50: 1514-1523.

9. Sabatine MS, Giugliano RP, Keech AC, Honarpour N, Wiviott SD, et al. (2017) Evolocumab and clinical outcomes in patients with cardiovascular disease. N Engl J Med 376: 1713-1722.

10. Schwarz GG, Szarek, Bhatt DL, Bittner V, Diaz R, et al. (2018) The Odyssey Outcomes Trial: topline results. Alirocumab in patients after acute coronary syndrome. Presented at: ACC 67th Scientific Sessions.

11. Zhang XL, Zhu QQ, Zhu L, Jian-Zhou Chen, Qin-Hua Chen, et al. (2015) Safety and efficacy of anti-PCSK9 antibodies: a meta-analysis of 25 randomized, controlled trials. BMC Med 13: 123.

12. Lloyd-Jones DM, Morris PB, Ballantyne CM, Birtcher KK, Daly DD Jr, et al. (2017) 2017 Focused Update of the 2016 ACC Expert Consensus Decision Pathway on the Role of Non-Statin Therapies for LDL-Cholesterol Lowering in the Management of Atherosclerotic Cardiovascular Disease Risk: A Report of the American College of Cardiology Task Force on Expert Consensus Decision Pathways. J Am Coll Cardiol 70: 1785-1822.

13. Arrieta A, Hong JC, Khera R, Virani SS, Krumholz HM, et al. (2017) Updated cost-effectiveness assessments of PCSK9 inhibitors from the perspectives of the health system and private payers: Insights derived from the FOURIER Trial. JAMA Cardiol 2: 1369-1374. 